\title{
Surgery for Massive Paediatric Head and Neck Neurofibroma: Two Case Reports
}

\author{
Asha'ari ZA', Kahairi $\mathrm{A}^{\mathrm{a}}$, Shahid $\mathrm{H}^{\mathrm{b}}$ \\ aDepartment of Otorhinolaryngology-Head \& Neck Surgery, Faculty of Medicine, International Islamic \\ University Malaysia, Jalan Hospital, 25100 Kuantan, Pahang, Malaysia \\ ${ }^{b}$ Department of Otorhinolaryngology-Head \& Neck Surgery, School of Medical Sciences, Hospital \\ Universiti Sains Malaysia, 16150 Kubang Kerian, Kelantan, Malaysia
}

\begin{abstract}
We report two paediatric patients with massive head and neck plexiform neurofibromas who underwent surgical excision. We wish to highlight issues and implications pertaining to the surgery, in particular, the management of intraoperative bleeding often encountered in these cases.
\end{abstract}

KEYWORDS: Plexiform neurofibroma, head and neck, surgery, paediatric, bleeding

\section{INTRODUCTION}

Neurofibromas are benign tumours of the peripheral nerves. Extensive head and neck neurofibromas in children often pose a surgical dilemma because of the higher risk of intraoperative complications, neurological deficits and recurrences. We report two children with massive deep-seated head and neck neurofibromas who underwent surgical excision and discuss concerns related to surgery in these cases.

\section{CASE 1}

A 12-year-old girl with a family history of neurofibromatosis type 1 presented with a slow growing swelling over the right side of her face for more than nine years. At the time of presentation, the tumour was extensive, involving the whole right side of the face, from the hairline superiorly until the midpoint of the neck inferiorly (Figure 1a). The right eye was alienated outwards and inferiorly, and she was blind in that eye.

Magnetic resonance imaging (MRI) showed a large, well-defined lobulated heterogeneous and nodular lesion, which covered the right side of her face and measured $22.5 \mathrm{~cm} \times 13.0 \mathrm{~cm} \times 15.5 \mathrm{~cm}$ (Figure $1 \mathrm{~b}$ ). The mass displaced the zygomatic arch laterally, displaced the globe anterior and inferiorly and extended via the right optic canal to the base of the

Corresponding author:

Dr Zamzil Amin Asha'ari

Associate Professor

Department of Otorhinolaryngology-Head \&

Neck Surgery,

Faculty of Medicine,

International Islamic University Malaysia,

Jalan Hospital, 25100 Kuantan, Pahang, Malaysia

Email: zamzilamin@yahoo.com

Phone: 006019-9564996 skull. Inferomedially, the mass extended into the right infratemporal and pterygopalatine fossa, crossing the midline to obliterate the right nasopharynx and oropharynx. Magnetic resonance angiography showed prominent feeding vessels supplying the tumour from branches of the external carotid artery. These vessels were embolised using fat tissue one day prior to the surgery. A diagnosis of neurofibroma was made on the basis of fine-needle tissue biopsy of the mass.

The patient underwent debulking of the tumour and right eye exenteration. Most of the tumour was removed, except for the tumour beneath the skin, which was preserved for primary closure. Despite preoperative embolisation, massive bleeding occurredduringtheexcision of thetumour, andwewere unable to satisfactorily control this bleeding with irrigation bipolar electrocautery. Bleeding was better controlled by applying a temporary vessel clamp on the external carotid artery, and the clamping was done intermittently throughout the surgery. The operation lasted for eight hours, and the blood loss was five litres. Extensive blood and fluid resuscitation were done intraoperatively and postoperatively in the ICU. Assessment at two weeks postoperatively showed that she had marked rightside facial weakness (Figure 1c). However, at the six-month follow-up, the weakness had improved to grade IV according to House and Brackmann's classification. ${ }^{1}$ Three years later, she underwent repeat MRI, which showed the recurrence of a small tumour in the right cheek region measuring $3.2 \times$ $8.0 \times 1.5 \mathrm{~cm}$. The patient declined subsequent operations to remove the tumour. 
Figures

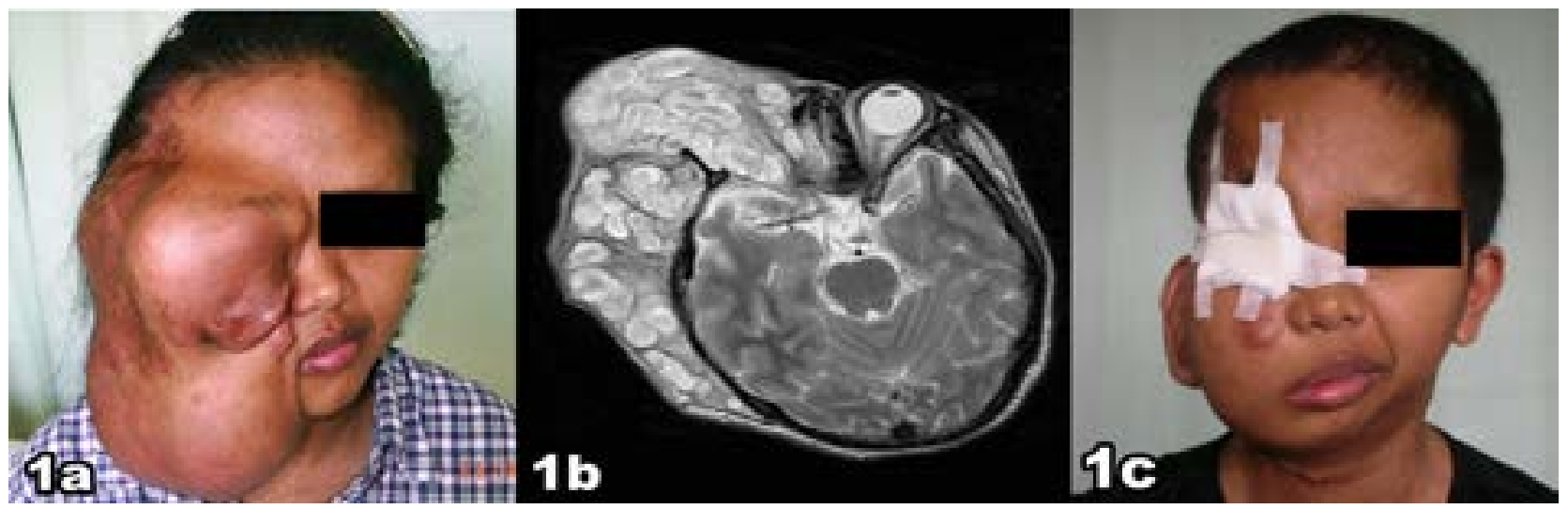

Figure 1.(a)Extensive plexiform neurofibroma with facial disfigurement; (b) axial T1-weighted MRI image showing extensive right orbital displacement; (c) ten-day post surgery

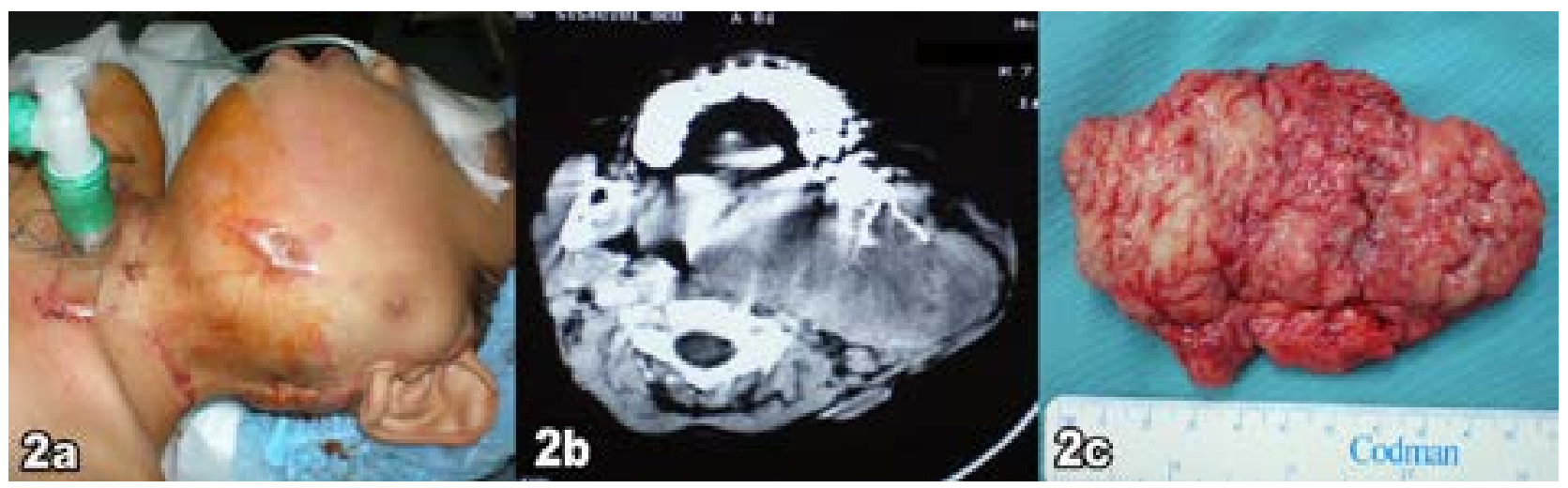

Figure 2.(a) Left parotid region swelling with scar tissue from a previous parotidectomy and biopsy;(b) axial CT image showing a solid mass in the left deep lobe of the parotid compressing the airway (arrow); (c) total tumour mass removed

\section{CASE 2}

A six-year-old boy with a history of left parotid swelling for some years presented with increasing breathlessness of three days duration. He had a left superficial parotidectomy two years earlier at another centre, and the histological diagnosis was a plexiform neurofibroma. A CT scan revealed a large solid mass in the left deep lobe of the parotid gland. The mass obliterated the left parapharyngeal space, leading to narrowing of the pharyngeal airway (Figure 2). A fine-needle aspiration biopsy of the mass confirmed the diagnosis of neurofibroma.

Due to increasing breathlessness, an emergency tracheostomy was done, together with debulking of the tumour. The incision was made at the old parotidectomy scar. Despite preoperative embolisation of the vascular supply to the tumour, it was difficult to stop bleeding from the bulk of the tumour itself and from the fibrotic tissues due to the prior surgery. The use of a harmonic scalpel for tissue dissection helped to minimise the blood loss. The bulk of the tumour was removed with the deep lobe of the parotid, and the histopathology showed no features of malignancy.
Thetracheostomywasdecannulatedontheseventh postoperative day. The patient was well six months postsurgery when he underwent another CT scan. This revealed tissue fibrosis of the left parotid and the parapharyngeal space, with a patent airway. He was seen again at one year follow-up and showed no clinical evidence of recurrence of the tumour.

\section{DISCUSSION}

It has been estimated that $25 \%$ of all cases of neurofibroma occur in the head and neck, ${ }^{2}$ with surgery as a primary treatment option for large plexiform neurofibromas. ${ }^{3}$ The indications for surgery are to diagnose malignant changes in a rapidly enlarging mass, to relieve pain and airway obstruction from compression of the tumour and to improve cosmetic outcomes in those with disfiguring diseases. ${ }^{4}$

Surgical management of massive plexiform neurofibroma of the head and neck must address the following main concerns: the timing of the surgery, the extent of the tumour that needs to be removed to balance the risk of recurrence with the loss of function and the risk 
of uncontrolled bleeding intraoperatively in large tumours. Younger age, the tumour's location in the head and neck region and incomplete surgical resection are predictive factors for a higher risk of the tumour progressing and recurring. ${ }^{5}$ Our two cases fell into these categories. Thus, recurrence was expected, particularly in case 1, where we were unable to totally remove the tumour. In such cases, lifelong follow-up is often warranted.

Neurofibromas are often richly supplied with inconspicuous, thin-walled abnormal feeding vessels. As a result, moderate to severe bleeding is a recognised feature of operations. The abnormal vascularity of the tumour and its infiltrative nature can cause difficulty in controlling the bleeding. Case series on surgery of large head and neck neurofibromas also reported bleeding as one of the main difficulties encountered intraoperatively. ${ }^{6,7}$ Extensive tumours may be associated with massive bleeding. Therefore, it is wise not to delay surgery in what is a progressive disease. Steps should be taken to minimise and control the anticipated intraoperative bleeding. This begins with the surgeons themselves: they must be experienced in performing oncological head and neck surgery and possess meticulous surgical dissection techniques. Hypotensive anaesthesia and an efficient resuscitation team in the operating theatre are also necessary to reduce blood loss during surgery. In addition, preoperative selective angiography and embolisation of the vascular supply to the tumour have an important role in reducing the bleeding. The traditional trans-arterial embolisation approach has proven beneficial, but it is sometimes limited by complex vascular anatomy and the tumour's location. Alternatively, direct puncture of head and neck tumours with an intralesional injection of acrylic glue or cyanoacrylate was found feasible and effective in achieving devascularisation of the tumour prior to surgery. ${ }^{8,9}$ When available, the use of modern surgical equipment like harmonic scalpels or irrigating bipolar electrocautery should reduce the bleeding rate intraoperatively. To minimise the blood loss, bipolar cauterisation of all bleeders, even the most minute, is recommended. The use of packing with haemostatic agents intraoperatively can help in securing the tumour's bleed, which is often a diffuse microcapillary bleed. Many materials have been used for packing such as oxidised cellulose polymer (surgicel), fibrin tissue glue, thromboplastin and collagen. A recent meta-analysis has shown strong evidence that tranexamic acid reduces blood transfusion perioperatively and that it can be recommended for massive vascular tumour surgery. ${ }^{10}$ The use of haemostatic medications, e.g. vitamin $\mathrm{K}$, vasopressin and somatostatin analogues, have been reported to be useful for reducing surgery-related bleeding, but there is a lack of evidence from randomised trials. ${ }^{11}$
Temporary compression of the external carotid artery by vascular clamping (Bulldog clamp) was helpful in case 1 to minimise the bleeding and is a recommended measure to help secure haemostasis in large tumour surgery. Clamping of the external carotid artery provides satisfactory control of bleeding during surgical procedures, without affecting the future blood supply. On the other hand, permanent ligation of the external carotid artery should be avoided, as this may result in the development of collateral circulation from the internal carotid and vertebral systems, which may complicate the control of bleeding during future operations. Unilateral external carotid artery clamping can be safely performed, without jeopardising blood supply to the head and neck. Clamping of the internal carotid artery is associated with a risk of stroke. Therefore, it is advisable to do the preoperative assessment for the risk of stroke during internal carotid artery occlusion. This consists of a selective four-vessel cerebral angiography, which establishes the patency of the cerebral vessels and the potential availability of collateral flow if one carotid artery is occluded. Intraoperative EEG monitoring is essential. If there is EEG evidence of ischaemic changes after crossclamping (slowing, voltage loss) of the internal carotid artery, the clamp should be released.

\section{CONCLUSION}

Bleeding is one of the main concerns in surgery of massive neurofibroma in the head and neck. Preoperative preparations, together with meticulous surgical techniques, can minimise bleeding. Temporary external carotid artery clamping helps to secure haemostasis when other measures have failed to control the bleeding.

\section{REFERENCES}

1. House JW, Brackmann DE. Facial nerve grading system. Otolaryngol Head Neck Surg 1985;93:146-7.

2. Marocchio LS, Oliveira DT, Pereira MC, Soares CT, Fleury RN. Sporadic and multiple neurofibromas in the head and neck region: a retrospective study of 33 33 years. Clin Oral Investig 2007;11:1659.

3. Packer RJ, Gutmann DH, Rubenstein $A$, et al. Plexiform neurofibromas in NF1: toward biologic-based therapy. Neurology 2002;58:1461-70.

4. Wise JB. Management of head and neck plexiform neurofibromas in pediatric patients with neurofibromatosis type 1 . Arch Otolaryngol Head Neck Surg 2005;131: 712-8.

5. Needle MN, Cnaan A, Dattilo J, et al. rognostic signs in the surgical 
management ofplexiform neurofibroma:

the Children's Hospital of Philadelphia

experience, 1974-1994. J Pediatr 1997;

131:678-82.

6. Manolidis S, Higuera S, Boyd V, Hollier LH.

Single-stage total and near-total resection of massive pediatric head and neck neurofi bromas. J Craniofac Surg 2006;17:506-10.

7. Rapado F, Simo R, Small M.

Neurofibromatosis type 1 of the head and neck: dilemmas in management. J Laryngol Otol 2001;115:151-4.

8. Pierot L, Boulin A, Castaings L, et al. Embolization by direct puncture of hypervascularized ORL tumors. Ann Otolaryngol Chir Cervicofac 1994;111: 403-409.

9. Abud DG, Mounayer C, Benndorf G, et al.Intratumoral injection of cyanoacrylate glue in head and neck paragangliomas. Am J Neuroradiol 2004;25(9):1457-62.

10. Ker K, Edwards P, Perel P, Shakur H, Roberts I. Effect of tranexamic acid on surgical bleed ing: systematic review and cumulative meta-analysis. BMJ 2012;17;344:e3054.

11. Erstad BL. Systemic hemostatic medications for reducing surgical blood loss. Ann Pharma cother 2001;35:925-934. 


\title{
Nasopharyngeal Carcinoma Presented with Right Upper Limb Weakness: A Case Report
}

\author{
Zamzuri Za, Mohd Adham SYa, Mohamad Saufi A ${ }^{\mathrm{b}}$, Azian $\mathrm{AA}^{\mathrm{c}}$, Mohamed Azril MA ${ }^{\mathrm{a}}$, Azlina $\mathrm{AR}^{\mathrm{d}}$ \\ a Department of Orthopaedics, Traumatology \& Rehabilitation, Kulliyyah of Medicine, International \\ Islamic University Malaysia, Kuantan, Pahang, Malaysia \\ ${ }^{b}$ Neurosurgical Division, Department of Surgery, Kulliyyah of Medicine, International Islamic University \\ Malaysia, Kuantan, Pahang, Malaysia \\ 'Department of Radiology, Kulliyyah of Medicine, International Islamic University Malaysia, Kuantan, \\ Pahang, Malaysia \\ dDepartment of Pathology, Hospital Tengku Ampuan Afzan, Kuantan, Pahang, Malaysia
}

\begin{abstract}
Nasopharyngeal carcinoma (NPC) is a malignant neoplasm arising from the mucosal epithelium of the nasopharynx, mainly within the lateral nasopharyngeal recess or Fossa of Rosenmuller. Distant metastasis to the cervical spine is the least common site in the vertebral system. This is a case of a 37-year-old lady with stage IV C nasopharyngeal carcinoma with local extension to the cervical spine who presented with right upper limb weakness.
\end{abstract}

KEYWORDS: Nasopharyngeal carcinoma, metastases, spine, upper limb

\section{INTRODUCTION}

Nasopharyngeal carcinoma (NPC) is a malignant neoplasm arising from the mucosal epithelium of the nasopharynx, mainly within the lateral nasopharyngeal recess or Fossa of Rosenmuller. The worldwide incidence of NPC is low $(<1$ per 100,000$)$ but it is one of the commonest malignancies among South East Asian Chinese and ranks as the second most common cancer among men in Malaysia. ${ }^{1,2}$ The most common presenting symptom is cervical lymphadenopathy, followed by nasal, aural and neurological symptoms from cranial nerve involvement. Distant metastases of nasopharyngeal carcinoma have been reported in the bones, lungs, liver, distant lymph nodes, brain and portal hepatis. The spine is the commonest site for bone metastases. The incidence of spinal metastases is likely to be increasing as is expected with increasingly older populations, longer life expectancy, and improvement in medical treatment.

\section{CASE REPORT}

A 37- year- old Chinese lady presented with weakness of the right upper limb for one-month. She had no history of trauma or exposure to tuberculosis. She

Corresponding author:

Dr Zamzuri Zakaria

Associate Professor,

Department of Orthopaedics, Traumatology \&

Rehabilitation,

Kulliyyah of Medicine,

International Islamic University Malaysia,

Jalan Hospital, 25150 Kuantan, Pahang, Malaysia

Tel: +60139906744

Fax: +6095144451

Email: zamdr@yahoo.co.uk had loss four kilograms of weight within a month. No history of tinnitus, reduced hearing or epistaxis complaint. The patient had unremarkable family and past medical history.

Cervical spine examination revealed paravertebral muscle spasm, moderate tenderness at the midcervical spine with a limited range of neck movement. There was a mass at right posterior triangle of the neck measuring $3 \times 2$ centimeters, smooth in surface, mobile, well defined margin and firm in consistency. There were no supraclavicular lymph nodes palpable. Neurological examination of the right upper limb revealed loss of shoulder abduction, reduced power of elbow flexion and extension to grade 2 . The reflexes were normal except for hyporeflexia of the right bicep. Sensation was reduced from $\mathrm{C} 4$ to $\mathrm{T} 2$ dermatome. The neurological examination of the left upper limb and both lower limbs including the cranial nerves were normal. The abdominal and breast examinations were unremarkable. She had a normal gait.

Her hemoglobin level was $14.4 \mathrm{~g} / \mathrm{dl}$ with normal white cell count. The erythrocyte sedimentation rate was $61 \mathrm{~mm} / \mathrm{hr}$, and the C-reactive protein was $26.5 \mathrm{mg} / \mathrm{L}$. The chest radiograph was normal. Plain radiograph of the cervical spine showed collapse of the $\mathrm{C} 5$ vertebra. Magnetic resonance imaging (MRI) of the cervical spine (Figure 1 \& 2 ) showed a mildly enhancing anterior and right paraspinal soft-tissue mass, which extended from the lower part of $\mathrm{C} 4$ to the upper part of $\mathrm{C} 6$ vertebral body measuring about $2.3 \times 2.8 \times 2.8 \mathrm{~cm}$. There was an extension to the $\mathrm{C} 5$ vertebral body, right facet joint and C4/C5 disc space. The mass also extended into the spinal cavity, obliterating the thecal sac and compressing on the spinal cord. Another soft-tissue mass was observed on the right side at the level of lower 
clivus to $\mathrm{C} 1$ measuring about $2.4 \times 4.7 \mathrm{~cm}$ causing obliteration of the larynx (Figure 3). Fibreoptic nasopharyngoscopy examination has detected fullness at the right fossa of Rosenmuller, and a biopsy was taken from there. The histopathology result revealed undifferentiated non keratinizing nasopharyngeal carcinoma. The diagnosis of nasopharyngeal carcinoma with metastases to the cervical spine (T3N1M1-stage IV C) was made.

The main indication for surgery in this patient was the progressive neurological deficit with evidence of spinal instability. She underwent 360 degree fixation combining anterior and posterior approach to the cervical spine, corpectomy of the C5 with discectomy of the C4,C5 and C5,C6 vertebra; and anterior fusion from C4-C6 augmented with an anterior plate. Lateral mass screw fixation was done from C3 to C7 vertebra. It was a one stage procedure with eight-hour duration of surgery. The amount of blood loss was 1.2 liters. Intraoperative finding was destruction of C5 vertebra body and facet joints with vascular soft tissue mass along the right paraspinal region of C4 and C5 vertebral bodies. Posteriorly there was destruction of the right C5 facet joint with vascularmassover the right C5lamina. Histopathology examination of the soft tissue was consistent with nasopharyngeal carcinoma (Figure 4).

LMM was referred to an oncology centre and was planned for a radical concurrent chemo-radiation. She underwent 35 cycles of radiotherapy and 7 cycles of chemotherapy with intravenous cisplastin. She responded well to the chemotherapy and radiotherapy. At follow-up six months post operatively, the right shoulder abduction improved to grade 4 and normal motor power for both elbow flexion and extension. There was no sensory improvement.

\section{Figures}

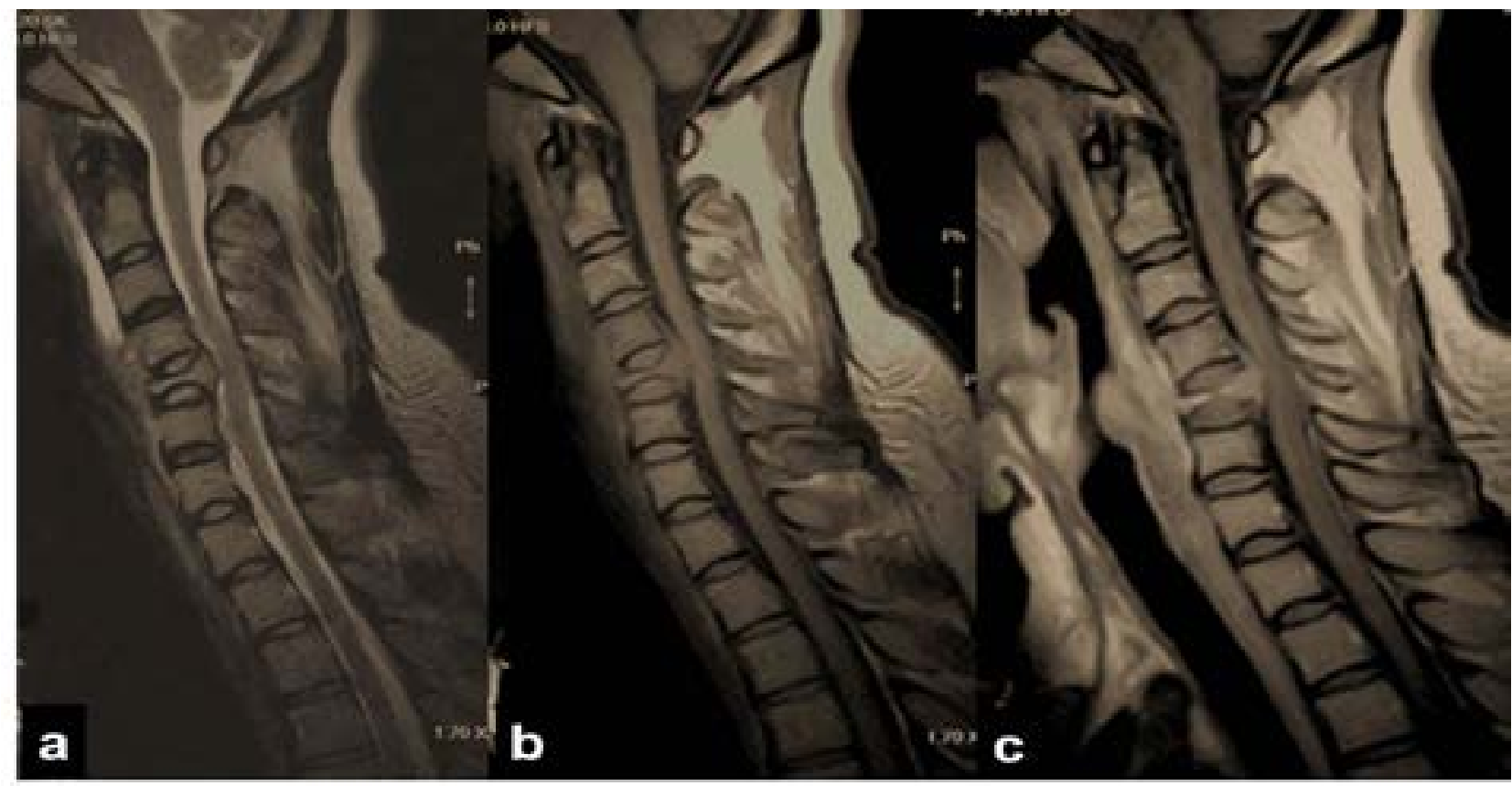

Figure 1: Sagittal MRI of Cervical spine - (a) T2, (b) T1, (c) Post IV Gad, demonstrating the collapsed C5 vertebra with obliteration of the anterior theca sac and compression onto the spinal cord by an enhancing soft tissue mass at that level

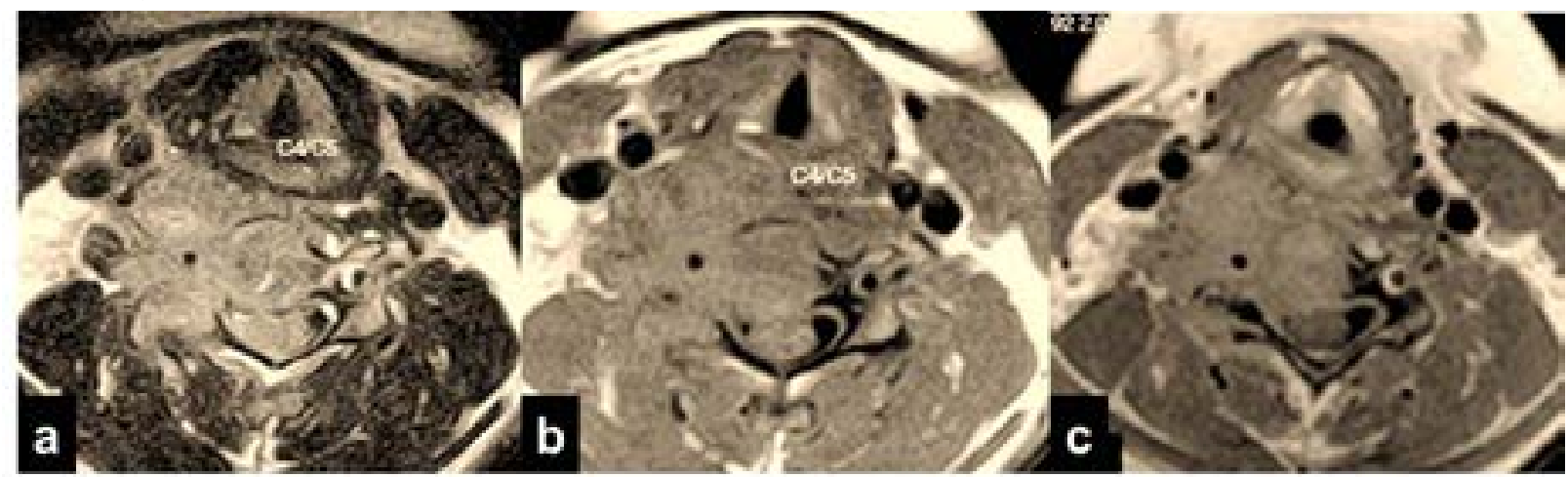

Figure 2: Axial MRI of Cervical spine - (a) T2, (b) T1, (c) Post IV Gad. A minimally enhancing soft tissue mass on the right side infiltrating the C5 vertebra and encasing the right neural foramen is shown. This mass extends into the spinal cavity obliterating the theca sac and compressing on the spinal cord. 


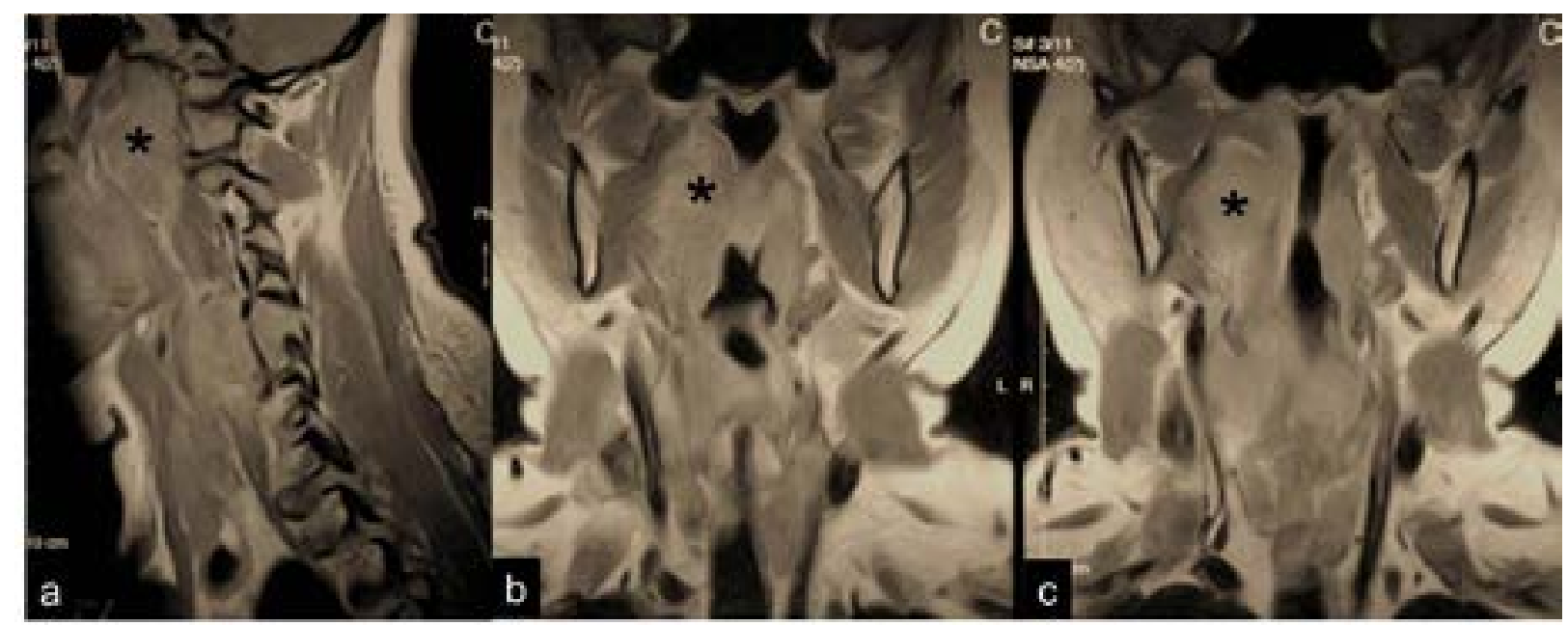

Figure 3: MRI of cervical spine post IV Gad. - (a) Sagittal, (b \& c) Coronal, showing a minimally enhancing soft tissue mass at the right nasopharynx (asterisk) in keeping with the HPE proven nasopharyngeal carcinoma.

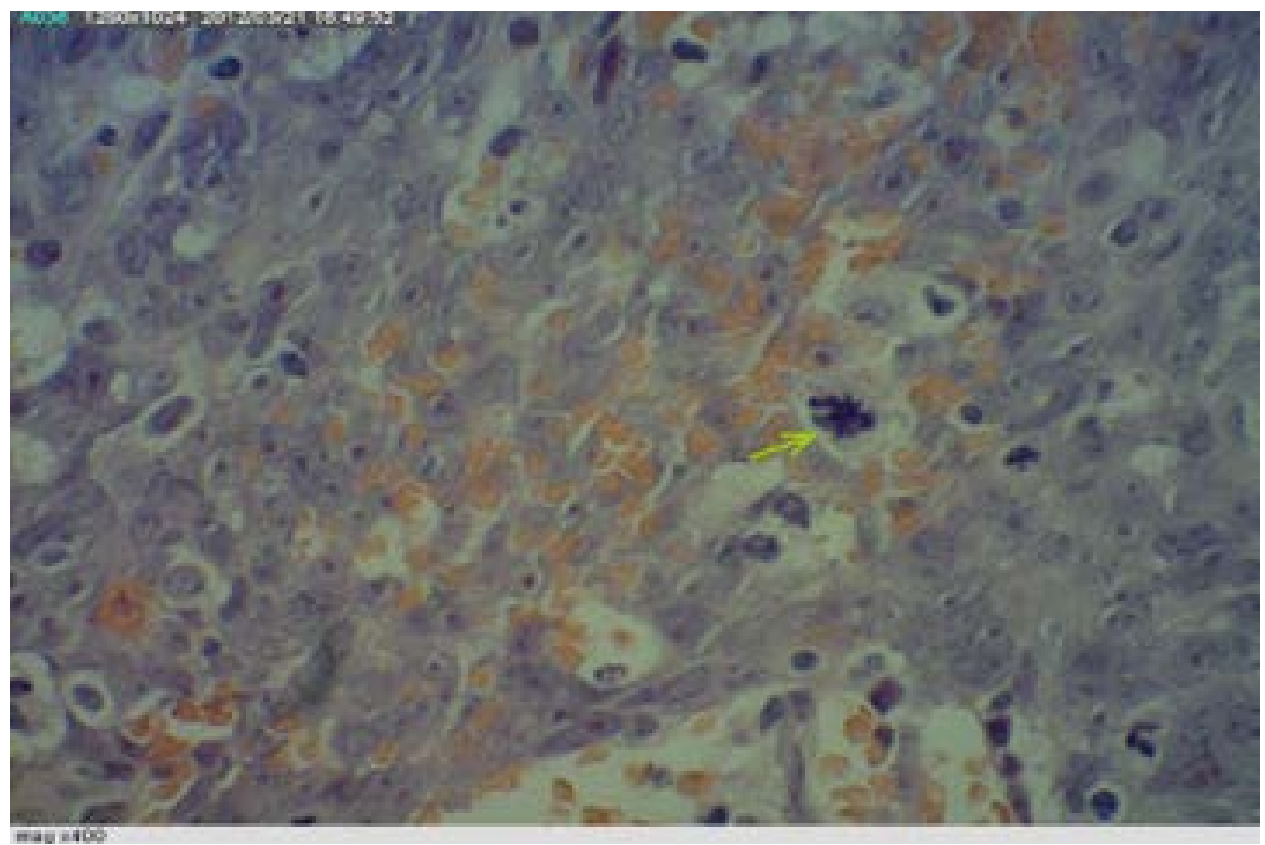

Figure 4: Individual tumour cells are in syncytial, consist of large, having oval nuclei, prominent eosinophilic nucleoli and frequent mitoses as shown by the arrow.

\section{DISCUSSION}

Our patient presented with right upper limb weakness due to local extension of the tumour toward the vertebral column and the spinal cord. As evidenced by the MRI findings, the tumour already involved the anterior, middle and the posterior column of the spine at presentation. It is very rare for NPC to present with solitary cervical metastasis. Even though the vertebral spine is a common site for skeletal metastases, only 3.5 percent of cases involved the cervical spine. ${ }^{3}$ There are few common symptoms observed in NPC patients. Swelling of the lymph nodes in the neck is the initial presentation in many patients. Symptoms related to primary tumour, which include hearing loss, otitis media, trismus and cranial nerve palsies have also been reported. Larger growths may produce nasal obstruction or bleeding, and metastatic spread may result in bone pain or organ dysfunction.
Staging of the NPC is mainly based on clinical and radiological examination. Our patient had a stage IV C disease which is considered as extensive neck disease with distant metastases.

The stage at presentation is the most significant prognostic factor. There are also other factors that may influence prognosis, which include the patient's age, gender, presence of keratinization, lymph nodes metastasis and genetic factor. Our patient had a poor prognosis as she presented with late-stage tumour (Stage IV C). The actuarial survival rate according to Ho's classification was reported to be 17.7 percent at 5 years and 9.5 percent at 10 years. ${ }^{4}$ The surgical treatment for our patient was mainly palliative, due to her poor prognosis. The surgicalstrategy forspinalmetastasesasproposedby Tomita et al. was adopted in her case. They 
proposed the indications for surgical intervention of metastatic spinal disease include: 1 ) intractable pain unresponsive to non-operative measures, 2) existence of a growing tumor that is resistant to radiation, chemotherapy or hormonal therapy, 3) spinal instability manifested as pathologic fracture, progressive deformity or neurologic deficit, and 4) clinically significant neural compression, especially by bone or bone debris. ${ }^{5}$ The main indications for surgery in this patient were progressive neurological deficit, evidence of spinal instability with significant neural compression based on the clinical and radiological findings. Even though the surgical treatment was mainly palliative, the important reason of combining the anterior and posterior fixation was mainly due to the fact that the tumor had already involved the anterior, middle and posterior column of the spine which may progress into further instability if it is not well stabilized surgically.

NPC is highly radiosensitive and thus radiotherapy (RT) is the mainstay treatment. In stage IV disease, radiotherapy with or without adjuvant chemotherapy, has been established as the standard of care based on multiple prospective randomized trials, ${ }^{6}$ as well as meta analyses which demonstrated a reduction of the risk of death by $18 \%$ and an increase in the 5 -year overall survival of $4-6 \% .^{7}$ It has been shown to provide significant improvements in both local control and distant metastases.

\section{REFERENCES}

1. Chan SH. Aetiology of nasopharyngeal carcinoma. Ann Acad Med Singapore 1990; 19:201-7.

2. Lim GCC, Halimah Y. Second report of the National Cancer Registry. Cancer Incidence in Malaysia 2003. Kuala Lumpur: National Cancer Registry, 2004.

3. Jonathan STS, Cheung YK, Chan D. Nasopharyngeal carcinoma: pattern of skeletal metastases. Br J Radiol 1990;63:202-5.

4. Teo PML, Leung SF, Yu P, et al. A comparison of Ho's, International Union against Cancer and American Joint Committee Stage Classification for nasopharyngeal carcinoma.

Cancer 1983;52:362-8.

5. Tomita K, Kawahara N, Kobayashi T, et al. Surgical strategy for spinal metastases. Spine 2001;26:298-306.

6. Wee J, Tan EH, Tai BC, et al. Randomized trial of radiotherapy versus concurrent chemoradiotherapy followed by adjuvant chemotherapy in patients with American Joint Committee on Cancer/International Union against cancer stage III and IV nasopharyngeal cancer of the endemic variety. J Clin Oncol 2005;23:6730-8.
7. Wei WI, Kwong DL. Current management strategy of nasopharyngeal carcinoma. Clin Exp Otorhinolaryngol 2010;3:1-12. 